\title{
Aberrant Striatal Value Representation in Huntington's Disease Gene Carriers 25 Years Before Onset
}

\author{
Akshay Nair, Eileanoir B. Johnson, Sarah Gregory, Katherine Osborne-Crowley, Paul Zeun, \\ Rachael I. Scahill, Jessica Lowe, Marina Papoutsi, Stefano Palminteri, Robb B. Rutledge, \\ Geraint Rees, and Sarah J. Tabrizi
}

\begin{abstract}
BACKGROUND: In this study, we asked whether differences in striatal activity during a reinforcement learning (RL) task with gain and loss domains could be one of the earliest functional imaging features associated with carrying the Huntington's disease (HD) gene. Based on previous work, we hypothesized that HD gene carriers would show either neural or behavioral asymmetry between gain and loss learning.

METHODS: We recruited $35 \mathrm{HD}$ gene carriers, expected to demonstrate onset of motor symptoms in an average of 26 years, and 35 well-matched gene-negative control subjects. Participants were placed in a functional magnetic resonance imaging scanner, where they completed an $\mathrm{RL}$ task in which they were required to learn to choose between abstract stimuli with the aim of gaining rewards and avoiding losses. Task behavior was modeled using an $\mathrm{RL}$ model, and variables from this model were used to probe functional magnetic resonance imaging data.

RESULTS: In comparison with well-matched control subjects, gene carriers more than 25 years from motor onset showed exaggerated striatal responses to gain-predicting stimuli compared with loss-predicting stimuli $(p=.002)$ in our RL task. Using computational analysis, we also found group differences in striatal representation of stimulus value $(p=.0004)$. We found no group differences in behavior, cognitive scores, or caudate volumes.

CONCLUSIONS: Behaviorally, gene carriers 9 years from predicted onset have been shown to learn better from gains than from losses. Our data suggest that a window exists in which HD-related functional neural changes are detectable long before associated behavioral change and 25 years before predicted motor onset. These represent the earliest functional imaging differences between HD gene carriers and control subjects.
\end{abstract}

https://doi.org/10.1016/j.bpsc.2020.12.015

Huntington's disease (HD) is a neurodegenerative disorder associated with a complex psychiatric, cognitive, and motor phenotype $(1,2)$. HD is caused by a CAG triplet repeat expansion in the huntingtin gene $(3,4)$. The length of this expansion is proportional to the age of onset of motor signs, which typically starts between 40 and 50 years of age (5). Although unequivocal motor features of HD are first seen at this point, neural atrophy and cognitive deficits are present earlier, in the premanifest phase (6-8). Given that diseaserelated changes occur before clinical diagnosis and with disease-modifying drugs for HD currently under investigation, an open question remains - what are the earliest changes associated with carrying the HD gene $(9,10)$ ?

$\mathrm{HD}$ is characterized by a range of cognitive deficits, particularly in fronto-executive functions such as attention, processing speed, set-shifting, and emotion recognition (11-13). Underlying these cognitive deficits is neuronal atrophy or dysfunction secondary to mutant huntingtin (mHTT) expression. In particular, the medium spiny neurons within the striatum, especially those within the indirect pathway, are highly susceptible to the HD disease process (14-16). In recent years, it has also become clear that the striatum is a key node in learning to maximize rewards (17-21). In keeping with these findings, differences in reward processing tasks have been found in persons carrying the HD gene (HDGCs), both in the manifest disease and in the premanifest phase up to 10 years before onset (22-26).

Learning to maximize rewards requires symmetrical performance in both learning to gain rewards and learning to avoid losses. Although these two processes involve different brain regions, both have been shown to activate the striatum (27-29). This is perhaps expected, as many computational models place the striatum at the center of behavioral policy adaptation that must occur in both pursuing gains and avoiding losses (30-32). Intriguingly, Palminteri et al. (23) reported in a behavioral study that premanifest HDGCs approximately 9 years from motor onset showed an asymmetry between gain and loss learning. In their study, premanifest HDGCs completed a reinforcement learning $(\mathrm{RL})$ task with both gain and loss domains. They found that premanifest 
HDGCs showed better learning from gains than from losses-a "reward bias." Computational modeling in this behavioral study further suggested that this effect was mediated by noisier decision making in the loss frame (23). Given the early involvement of the striatal indirect pathway in HD and the proposed role this pathway plays in learning from losses, such an asymmetry between gain and loss learning may be predicted as an early change associated with carrying the HD gene $(14,31,33-37)$.

On this basis, we asked whether a reward bias could be one of the earliest functional imaging features associated with carrying the HD gene. To address this question, we undertook a functional magnetic resonance imaging (fMRI) study in which young healthy HDGCs who were predicted, based on CAG length and age, to be 25 years from clinical diagnosis completed an $\mathrm{RL}$ task to gain rewards and avoid losses. We asked whether there was evidence of an asymmetry between gain and loss learning in the HDGCs, compared with wellmatched control participants, either at a behavioral level or in the corresponding fMRI signal. We looked for such a reward bias in frontostriatal regions of interest (ROls) at both cue and outcome presentation and used computational modeling to better understand our findings.

\section{METHODS AND MATERIALS}

\section{Subject Details}

We recruited 35 HDGCs and 35 matched control subjects who did not carry the HD gene. All participants were between 18 and 40 years of age. HDGCs were required to show no diagnostic motor features of HD (diagnostic classification score $<4$ ), have a CAG repeat length $\geq 40$, and have a disease burden score $\leq 240$, indicating that these patients are estimated to be at least 20 years from onset of motor symptoms. Control participants were required to have no known family history of HD or to have been tested for HD. Exclusion criteria were substance abuse, the use of any medications to treat HD, unstable dose of antidepressants in the past 30 days, and significant medical or psychiatric disorder. Groups were matched for age, sex, highest education level, and handedness. Demographics for both groups can be found in Table 1 . This study was a separate substudy within the Huntington's Disease Young Adults Study and was approved by the Bloomsbury Research ethics committee (16/LO/1323) (38).

\section{Task Description}

The task used in this study was identical in design to that described by Pessiglione et al. (29) and Palminteri et al. (23) to allow for meaningful comparison between results (see Figure S1). Participants completed an RL task in which the aim was to maximize rewards by learning to choose the best symbol from a pair of abstract symbols displayed on the screen. Participants saw 3 pairs of symbols, corresponding to the 3 conditions-gain, neutral, and loss. In each condition, one symbol was associated with an outcome with probability of 0.8 and the other symbol was associated with the same outcome with probability of only 0.2 . In the gain condition, this outcome was winning a fictional $£ 1$, signified by an image of a $£ 1$ coin with a green surrounding halo. In the loss condition, this outcome was losing a fictional $£ 1$, signified by an image of a $£ 1$ coin with a red cross superimposed over it. In both conditions, the alternative outcome was to receive nothing, signified by the word "Nothing" appearing on-screen. In the neutral condition, the two outcomes were either an empty gray disc or the word "Nothing" signifying no reward. For details of fMRI task presentation, see Supplemental Methods.

Participants were shown each pair of stimuli 30 times, with a total of 90 choices per run. Participants were given instructions and carried out 1 run of the task outside of the scanner to familiarize themselves with the task. Participants completed 2 runs in the scanner, with each run lasting on average 12 to 14 minutes. In the scanner, instructions were repeated before the task began.

\section{Behavioral and Computational Analysis}

Behavior from both runs was concatenated, and an RL model of behavior was fit to the subject data across the 2 runs.

Table 1. Sample Demographics

\begin{tabular}{|c|c|c|c|}
\hline & HD Gene Positive, $n=35$ & HD Gene Negative, $n=35$ & $p$ Value \\
\hline Age, Years & $29.4(5.7)$ & $30.5(5.2)$ & $.41^{a}$ \\
\hline Sex, Female, $n$ & 19 & 20 & $.81^{b}$ \\
\hline Handedness, Right, $n$ & 30 & 32 & $.70^{b}$ \\
\hline NART & $102.2(6.9)$ & $103.4(8.3)$ & $.52^{a}$ \\
\hline Depressive Score & $32.2(8.6)$ & $34.6(7.4)$ & $.22^{a}$ \\
\hline Adjusted Caudate Volume, $\mathrm{mL}$ & $7.27(0.75)$ & $7.42(0.67)$ & $.40^{a}$ \\
\hline UHDRS Motor Score, Median (Maximum) & $0(5)$ & & \\
\hline CAG Repeat Length & $41.8(1.2)$ & & \\
\hline Disease Burden Score & $185.1(33.5)$ & & \\
\hline
\end{tabular}

Values are reported as mean (SD) unless otherwise stated.

Sample demographics for both groups show that the cohorts were well matched at recruitment for age, sex, and handedness. Groups were matched for intelligence, as measured by the NART, and for depressive symptoms, as measured on the Zung Depressive Scale. Groups were in the normal range for depressive symptoms $(<50)$. Caudate volumes adjusted for total intracranial volume (see Methods and Materials) were also not significantly different between groups.

HD, Huntington's disease; NART, National Adult Reading Test; UHDRS, Unified Huntington's Disease Rating Scale.

The $p$ value was calculated via independent $t$ test.

${ }^{b}$ The $p$ value was calculated via $\chi^{2}$ test. 
Percentage correct was determined by the number of times a subject chose the best symbol in the gain and loss pairs. The difference between the percentage correct in gains and losses was used to compute the reward bias term. Differences between groups were tested using either rank sum tests or independent $t$ tests, where appropriate, after assessment of data distribution, with $z$ and $t$ statistics reported, respectively. In all tests, $n=35$ in each group. All behavioral analyses were completed in MATLAB R2017a (The MathWorks, Inc., Natick, MA).

Behavior was modeled using a Q-learning model, combining a Rescorla-Wagner learning model with a softmax action selection mechanism. The value of the chosen option $(Q)$ was updated for the next trial $(i+1)$ by updating its current estimated value with the prediction error $(\delta)$ multiplied by the learning rate $(\alpha)(39)$ :

$$
Q_{a(i+1)}=Q_{a(i)}+\alpha * \delta_{(i)}
$$

The prediction error term was calculated as follows:

$$
\delta_{(i)}=\operatorname{reward}_{(i)}-Q_{a(i)}
$$

where reward $_{(i)}$ represents the outcome following choice: +1 for gaining money, 0 for nothing, and -1 for losing money. Action selection was modeled using the softmax function, in which the probability of choosing an action is determined as follows:

$$
P_{a}(t)=\frac{e^{Q_{a}(t) * \beta}}{\sum_{i=1}^{n} e^{Q_{i}(t) * \beta}}
$$

Using this action selection rule, the probability that an action is chosen is based both on its relative value to the other option and on a computational term, choice temperature $(\beta)$, which represents choice stochasticity. Based on model comparison, initial $Q$ values for both symbols for gain domain choices were set at 0.5 and -0.5 for loss domain. This model provided a good fit for both gains and losses, with no difference of fits between groups, and it performed best in terms of model comparison across 6 competing models. For details on model fitting, see Supplemental Methods and Figure S2.

\section{MRI Acquisition}

Alongside acquisition of a T1-weighted sequence and field maps, functional imaging data were collected using a standard 2-dimensional echo-planar imaging sequence on a $3 T$ Siemens Prisma system (Erlangen, Germany). A sequence optimized to minimize drop-out in regions near the striatum, orbitofrontal cortex, and amygdala was used (40). For details on MRI acquisition and structural image processing, please see Supplemental Methods.

\section{Functional Imaging Preprocessing and Modeling}

Standard echo-planar imaging preprocessing steps were taken; see Supplemental Methods. At the participant level, a general linear model (GLM) was constructed, with cue onsets and outcome onsets as regressors of interest. Cue onsets were subdivided by valence and whether the button was pressed (i.e., regressors for gain cues were Gain-Go and Gain-
NoGo). A second GLM was built for each subject for the computational model-based fMRI analysis containing the cue and reward onsets. The model-derived $Q$ values and reward prediction error (RPE) from the computational model above were then used as parametric modulators at the time of choice and outcome, respectively. As no group difference in parameter estimates was found (see Results), individual $Q$-value regressors and RPE regressors were estimated from each subject's choice behavior using the mean learning rate parameters across the whole group $\left(n=70, \alpha_{\text {gain }}=.122, \alpha_{\text {loss }}=\right.$ .220). This fixed-effects procedure has been used in multiple previous studies and is argued to be a more robust approach for computational fMRI (41-43).

At the second level, GLMs including group membership alongside covariates of age, sex, handedness, adjusted caudate volumes, and depression scores were estimated for model-free and model-based contrasts specified at the first level. The results of these second-level GLMs were imported into the MarsBaR for ROI analysis in which mean GLM parameter $(\beta)$ estimates within regions of interest were used to either confirm replication of previous results or test our hypotheses.

\section{ROI Approach for Replication Analysis}

To confirm replication of previously reported results using this task, we assessed the significance of parameter estimates within spherical ROls defined from previously reported results. We created 6-mm spherical masks seeded at $[ \pm 12,10,-10]$ for the left and right ventral striatum, $[ \pm 30,28,-6]$ for the bilateral insula, and $[-1,27,-18]$ for the medial prefrontal cortex $(29,44)$. Based on previous work, we assessed activity in these regions for the contrasts gain cue $>$ neutral cue, loss cue $>$ neutral cue, win money $>$ lose money, lose money $>$ win money. We also assessed activity in these regions for $Q$ values and RPE. No exploratory whole-brain group differences were assessed.

\section{ROI for Reward Bias Analysis}

To restrict our search for group differences to relevant brain regions, we first sought to determine regions that showed a reward bias in our task. Unbiased ROls were derived from shared activity across both groups using the contrasts winning money $>$ losing money or gain-predicting cues $>$ losspredicting cues after whole-brain voxelwise correction at familywise error $p<.05$ with a minimum cluster extent of 10 . These voxels showed increased activity for rewards or rewardpredicting cues compared with that for losses or losspredicting cues. Clusters in the left and right striatum and prefrontal cortex were identified. These clusters were extracted using the MarsBAR toolbox in SPM. Striatal clusters were bounded by Automated Anatomical Labelling Atlas masks for the left and right caudate (see Figure S3). Previous work both in patients with HD and with this task suggests lateralization of the fMRI signal, and so left and right striatal clusters were kept separate $(24,29)$.

Second-level GLMs were imported into MarsBAR, and average parameter $(\beta)$ values within each $\mathrm{ROI}$ were estimated by subject. To test our hypothesis, gain cue $>$ loss cue and the win money $>$ lose money contrasts were compared between 
groups, using the contrast HDGCs > control subjects, correcting for age, sex, handedness, adjusted caudate volume, and depression scores. For each of these contrasts, differences were assessed in $3 \mathrm{ROls}$ (left and right ventral striatum and medial prefrontal cortex). As such, we tested for a difference between our groups using 2 contrasts, one at cue presentation and the other at outcome presentation, across 3 ROls. To correct for this multiple comparison across these 6 texts, a stringent Bonferroni threshold of $p<.008$ was considered significant. Confirmatory analysis was then performed using only the appropriate 6-mm spherical ROls defined from the literature as described above.

Model-based $\mathrm{fMRI}$ analysis was then used to assess whether neural correlates of $Q$ value or RPE error differed by group. As above, analyses were performed in MarsBAR using the contrast HDGCs > control subjects for each of the $3 \mathrm{ROIs,}$ with a threshold of $p<.008$ considered significant.

\section{Data Availability}

Data will be shared on reasonable request post publication in accordance with Wellcome Trust open access data-sharing policy.

\section{RESULTS}

We recruited 35 HDGCs and 35 closely matched HD genenegative control subjects. There were no differences in National Adult Reading Test scores, current depressive symptoms (Table 1 and Figure S4), or core cognitive tests typically sensitive to HD: Stroop word $(t=-1.33, p=.19)$ and Stroop color reading $(t=-1.06, p=.29)$, Symbol Digit Modalities Test $(t=-0.43, p=.67)$, and verbal fluency $(t=-1.58, p=.12)$. No significant caudate atrophy, typically a highly sensitive marker of HD (15), was seen in the HDGC group (Table 1 and Figure S5). The HDGCs had an average CAG length of 41.8 ( \pm 5.7$)$, giving a mean estimated years to onset of 26.1 years ( \pm 5.5 years) based on the established Langbehn formula (5). The median unified HD motor score in this group was 0 , with a maximum of 5 , indicating that this group was definitely premanifest.

\section{Task Performance}

Participants completed an RL task in which they had to learn to choose between pairs of abstract stimuli associated with rewarding and punishing outcomes, as shown in Figure S1 $(23,29)$. Both groups learned to choose the most rewarding symbol and avoid the most punishing symbol (Figure 1A, B). Both groups also showed more correct responses for gains than losses (gene carriers: $t=3.35, p=.002$; gene negative: $t=$ 2.27, $p=.03$ ). However, this behavioral reward bias was not significantly different between the groups $(t=1.19, p=.28)$ (Figure 1C). There was no significant difference in the percentage correct in either gains or losses between groups $\left(z_{\text {gains }}=-0.11, p_{\text {gains }}=.91, z_{\text {losses }}=-.73, p_{\text {losses }}=.46\right)$ (Figure 1D).

\section{Computational Modeling of Behavior}

A Q-learning model fit behavior well in both groups (Figure 1A, B). Mean fitted groupwise parameter estimates for the learning rate and choice temperature from this model are shown in Figure 2. There was no significant parameter difference between the groups in either gains or losses $\left(\alpha_{\text {gain }}: z=-1.22\right.$, $p=.22 ; \beta_{\text {gain }}: z=-0.41, p=.68 ; \alpha_{\text {loss }}: z=-1.29, p=.19$; $\beta_{\text {loss: }}: z=-0.47, p=.20$ ).
A

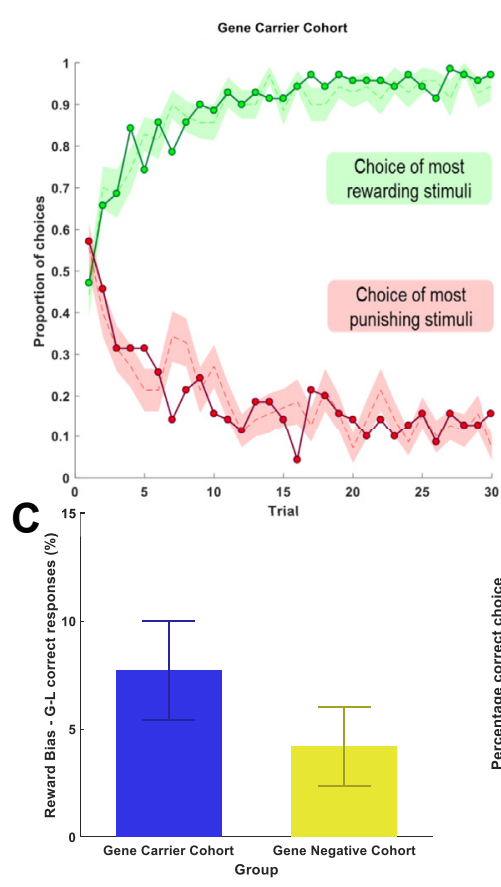

B

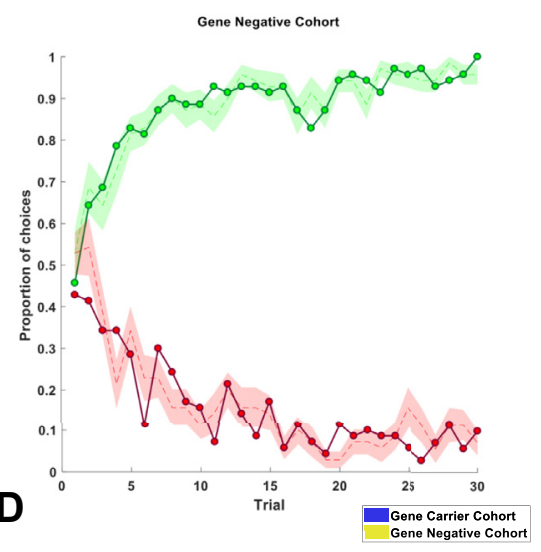

Figure 1. Both groups performed the task well, with no group differences. Participants in the (A) HDGC $(n=35)$ and (B) control $(n=35)$ groups learned to choose the most rewarding symbol in the gain $(\mathrm{G})$ trials and avoid the most punishing symbol in the loss (L) trials. Mean participant choice data are shown by the dashed lines, with shaded regions showing \pm SEM, green for gains and red for losses. The connected dotted green and red lines show mean computational model performance for gains and losses, respectively, showing computational modelpredicted behavior well in both groups. (C) Both groups showed marginally better performance in learning from gains than in learning from losses with small positive reward bias (mean percentage correct in gains minus loss). (D) Percentage correct in gains and losses by group. Bars show mean correct, with error bar showing \pm SEM. No significant group difference was observed in these metrics. 


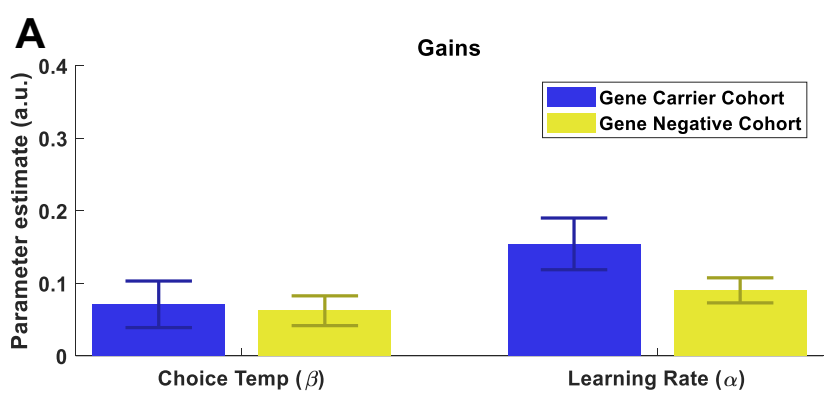

B

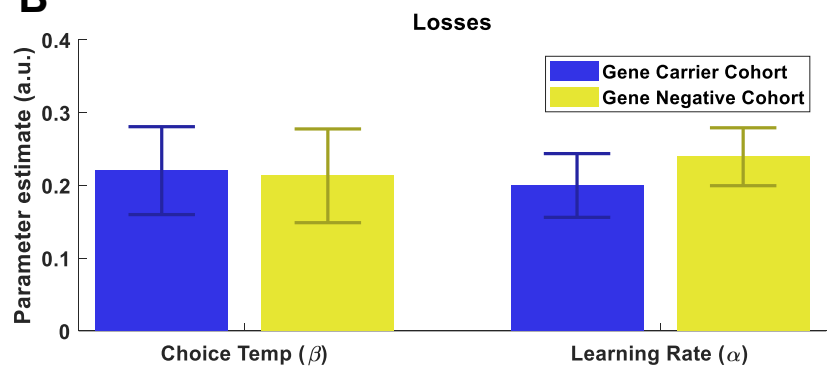

Figure 2. Computational model parameter estimates by group and valence. Mean parameter estimates for $Q$-learning model ( \pm SEM) for each group in (A) gains and (B) losses-no significant group difference was observed. a.u., arbitrary units.

\section{Robust Activation of Frontostriatal Regions Associated With Task}

As shown in Tables 2 and 3, we replicated, using spherical ROls defined from existing literature, canonical neural findings associated with performance in an $\mathrm{RL}$ task. Bilateral ventral striatum (VS) activity was seen in response to gain and loss cues compared with neutral cues, reflecting learned stimulus value and associated with the RPE. Medial prefrontal cortex (mPFC) activity correlated with positive stimulus valence, the $Q$-value regressor, and RPE. Bilateral insula activation was associated with loss cue onset and the inverse, or punishment, prediction error. Figure 3 (and Figure S6) shows task activity at the whole-brain level across both groups at a liberal threshold, for illustration purposes only.

\section{Evidence of Ventral Striatal Reward Bias at Cue Onset in HDGCs}

We identified clusters in the left and right VS and mPFC, stringently defined and unbiased with respect to group, that showed a reward bias in our task (see Methods and Materials) (ROls are shown in Figure S3).

After adjusting for covariates of age, sex, handedness, adjusted caudate volume, and depression scores, we assessed whether mean parameter estimates in these regions were greater in HDGCs than in control subjects using 2 reward bias contrasts. Firstly, at cue presentation we compared gain cue presentation with loss cue presentation. Likewise, at outcome presentation we compared activity for winning and losing money. More positive estimates in these contrasts would suggest an asymmetry between gain and loss learning. To account for testing these 2 contrasts in our 3 ROls, we used a stringent Bonferroni threshold of $p<.008$.
At cue presentation, we found that the HDGC group, compared with the control subjects, showed an enhanced left VS response comparing gain cue presentation with loss cue presentations $\left(t=2.98, p_{\text {uncorrected }}=.002\right)$. This effect was not seen in the right VS $\left(t=0.69, p_{\text {uncorrected }}=.25\right)$ or the $\mathrm{mPFC}(t=$ $\left.0.68, p_{\text {uncorrected }}=.25\right)$. Individual subject parameter estimates by group are shown in Figure 4A. To ensure that these results were not influenced by the choice of ROI, a 6-mm spherical $\mathrm{ROI}$ was created in the left VS at the coordinates described by Pessiglione et al. (29), and results showed the same difference between groups ( $t=2.36, p=.01$ )

No significant difference between groups was seen at outcome presentation comparing winning money to losing money in any of the 3 ROls (left VS: $t=1.07, p=.14$; right VS: $t=1.03, p=.16$; mPFC: $t=0.40, p=.35$ ).

\section{Ventral Striatal Reward Bias Seen in Striatal Response to Model-Derived Value Estimates}

In a complementary analysis, we compared activity in our 3 ROls using regressors extracted from the computational model, the $Q$ value, and the RPE $(\delta)$. Here, we use these regressors as complementary probes to the model-free fMRI analysis. Both regressors are positive in gain conditions and negative in loss conditions. More positive parameter estimates reflect a wider difference in neural activity between gain and loss domains in the cue and outcome phases of the task, for $Q$ value and RPE, respectively. As above, we hypothesized that this would occur in the HDGC group compared with control subjects, and as before, we considered a Bonferroni threshold of $p<.008$ to be significant. As before, we adjusted this analysis for covariates of age, sex, handedness, adjusted caudate volume, and depression scores.

In keeping with the model-free analysis, we found that the HDGC cohort compared with the control subjects had higher parameter estimates associated with the $Q$-value regressor in the left VS $\left(t=3.56, p_{\text {uncorrected }}=.0004\right)$ but not the right VS $(t=$ $\left.0.82, p_{\text {uncorrected }}=.21\right)$ or the medial prefrontal cortex $(t=1.14$, $\left.p_{\text {uncorrected }}=.13\right)$. Using the independent spherical left VS ROI, we found the same differences between groups $(t=2.69, p=$ .005). Individual parameter estimates by group are shown in Figure 4B. We found no evidence of a difference in the $\beta$ values associated with RPE-related activity in the HDGC cohort (left VS: $t=-0.1, p=.54$; right VS: $t=-0.34, p=.63$; mPFC: $t=-0.46, p=.68$ ).

Neither the parameter estimates in the gain versus loss contrast $(r=-.03, p=.89)$ nor the $Q$-value parameter estimates $(r=-.11, p=.53)$ in the HDGC group correlated with the disease burden score.

\section{More Robust Response to Loss Cues in Control Subjects}

To display the reward bias described above, either HDGCs should show a greater response to gain cues than control subjects or the control subjects should display greater response to loss cues than HDGCs, or both. Post hoc, using the unbiased task-defined left VS ROI, we tested these hypotheses by comparing valence cue onsets with neutral cue onsets. HDGCs did not show an enhanced striatal response comparing gain cues with neutral cues $\left(t=0.95, p_{\text {uncorrected }}=.17\right.$ ); however, 
Table 2. Replication Analysis at Cue Presentation Across HD Gene-Positive and HD Gene-Negative Groups

\begin{tabular}{|c|c|c|c|c|c|c|}
\hline & \multicolumn{2}{|c|}{ Gain $>$ Neutral Cue } & \multicolumn{2}{|c|}{ Loss $>$ Neutral Cue } & \multicolumn{2}{|c|}{$Q$ Value } \\
\hline & $t$ & $p$ & $t$ & $p$ & $t$ & $p$ \\
\hline Left VS & 3.61 & $<.001$ & 4.76 & $<.001$ & -0.018 & .5 \\
\hline Right VS & 5.77 & $<.001$ & 6.77 & $<.001$ & -0.014 & .51 \\
\hline Medial PFC & 2.75 & $<.004$ & -1.71 & .95 & 5.61 & $<.001$ \\
\hline Bilateral Insula & 1.22 & .11 & 6.33 & $<.001$ & Not tested & Not tested \\
\hline
\end{tabular}

$N=70$.

The table describes the 1 -sided $t$ statistic and corresponding $p$ value whether activity in regions of interest, defined from existing literature, was positively associated with contrast at cue presentation.

HD, Huntington's disease; PFC, prefrontal cortex; VS, ventral striatum.

control subjects did show significantly increased response to loss cues than neutral cues $\left(t=2.03, p_{\text {uncorrected }}=.02\right)$.

\section{DISCUSSION}

In this study, we recruited a cohort of healthy HDGCs estimated to be 25 years from onset of motor symptoms, based on age and CAG length, and found that neural activity in response to stimulus valence and value was significantly different from matched control subjects in the striatum. No group difference was seen in behavior, cognitive scores, or caudate volumes. Taken together, these findings suggest that changes in the frontostriatal value networks may occur very early in the life of HDGCs; however, these changes are not sufficient to manifest as behavioral differences, 25 years before motor onset. To our knowledge, our findings are the earliest reported functional imaging differences between HDGCs and control subjects.

In a behavioral study, Palminteri et al. (23) reported an asymmetry between gain and loss learning, a reward bias, in HDGCs approximately 10 years from motor onset. Although they did not collect fMRI data, they found that these behavioral differences may be driven by computational differences at the choice phase, with HDGCs making noisier decisions in the loss frame. These findings are wholly in keeping with our fMRI findings. We also found a difference between our groups at the choice phase with some evidence that this difference is mediated by impaired loss cue representation in the VS. We found no difference at outcome or in our behavioral analysis. As both studies used the same task, the neural changes identified here may be antecedent to the behavioral and computational changes identified by Palminteri et al. (23) and may be predicted to occur in our cohort in approximately 15 years. These findings also raise three further possibilities. Firstly, they suggest that a window exists in which functional neural changes occur in HDGCs, before measurable atrophy and corresponding behavioral changes. Secondly, these findings may be in keeping with early dysfunction of the indirect pathway in HDGCs, which is lost at a greater rate in HD and thought to contribute to loss learning and avoidance (14,31,33,35-37). Finally, this study adds to the growing body of evidence that suggests that limbic and reward processing may be among the most sensitive and early cognitive markers of $\operatorname{HD}(8,23,24,45,46)$.

It has been hypothesized, secondary to disinhibition resulting from the loss of striatal medium spiny neurons, that dopaminergic signaling in HD follows an inverted U-shape in which low levels of dopamine are found later in HD whereas increased dopamine signaling is found earlier in the disease (47). In keeping with this model, using a reversal learning task, Nickchen et al. (22) reported the loss of RPE signaling in the left VS of patients with manifest HD, especially those more severely affected. Pharmacological induction of a hyperdopaminergic state using our task has been shown to enhance RPE signaling; however, we found no difference between our HDGC group and control subjects in striatal RPE activity to suggest exaggerated dopamine signaling $(29,48)$. It may be that participants in our HDGC group were too far from onset for dopamine dysregulation to manifest as measurable differences in blood oxygen level-dependent activity. Similar to our results, in a nonlearning task with gain and loss domains, Enzi et al. (24) reported that left ventral striatal activity in HDGCs 10 years from onset showed impaired striatal representation of punishment cues in the left VS. Unlike our study, they did not find differences between HDGCs far from onset and control subjects. Aside from differences in sample size, these findings suggest that learning tasks may be more sensitive in very premanifest HD.

Table 3. Replication Analysis at Outcome Presentation Across HD Gene-Positive and HD Gene-Negative Groups

\begin{tabular}{|c|c|c|c|c|c|c|}
\hline & \multicolumn{2}{|c|}{ Win $>$ Lose Money } & \multicolumn{2}{|c|}{ Lose $>$ Win Money } & \multicolumn{2}{|c|}{ Reward Prediction Error } \\
\hline & $t$ & $p$ & $t$ & $p$ & $t$ & $p$ \\
\hline Left VS & 5.67 & $<.001$ & Not tested & Not tested & 3.96 & .001 \\
\hline Right VS & 6.19 & $<.001$ & Not tested & Not tested & 3.37 & .006 \\
\hline Medial PFC & 5.52 & $<.001$ & Not tested & Not tested & 5.54 & $<.001$ \\
\hline Bilateral Insula & Not tested & Not tested & 5.47 & $<.001$ & -6.02 & $<.001^{a}$ \\
\hline
\end{tabular}

$N=70$.

The table describes the 1 -sided $t$ statistic and corresponding $p$ value testing whether activity in regions of interest, defined from existing literature, was positively associated with contrast at outcome presentation.

HD, Huntington's disease; PFC, prefrontal cortex; VS, ventral striatum.

ansula activity was positively associated with the punishment prediction error. 


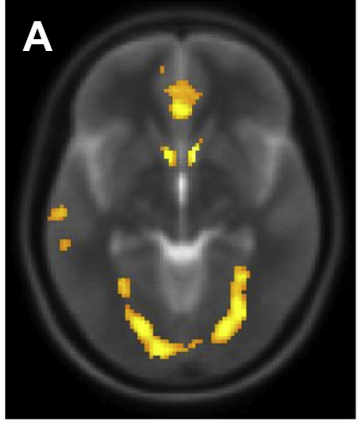

Gain - Neutral Cue Presentation

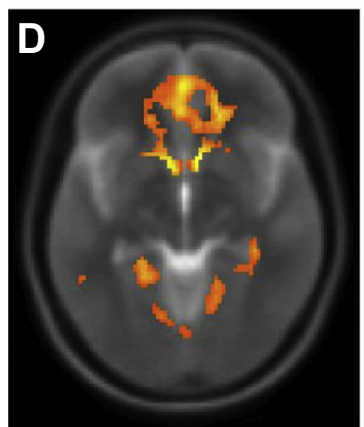

Winning - Losing

Money

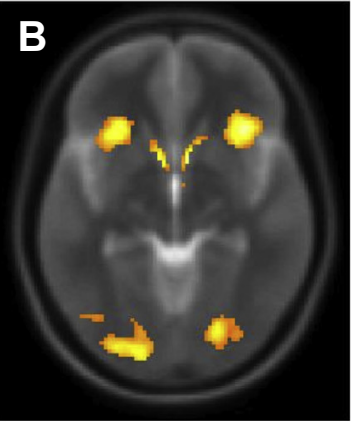

Loss - Neutral Cue Presentation

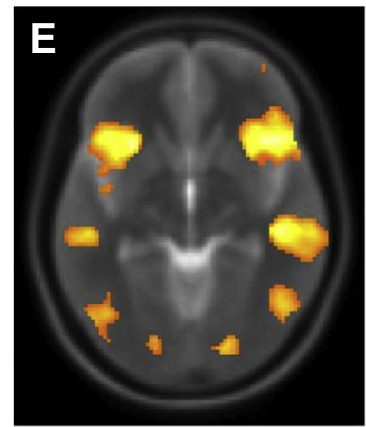

Losing - Winning Money

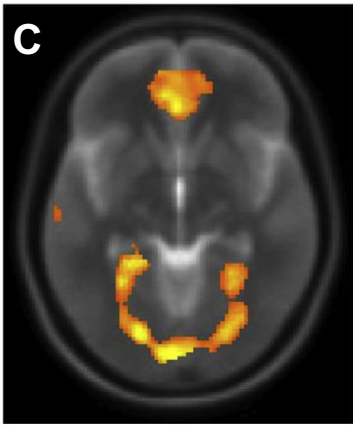

Q-value of Chosen Option

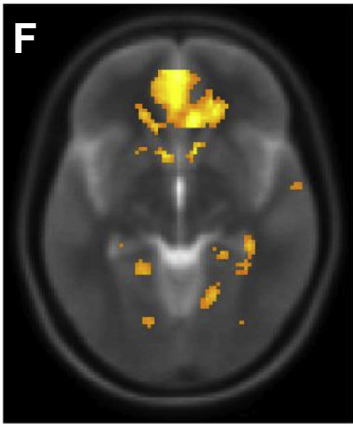

Reward Prediction Error

Figure 3. Neural activation in response to (A-C) choice and (D-F) outcome in task, for illustration purposes only. Neural activation (A-C) at cue presentation and (D-F) at outcome presentation across all subjects shown in the axial plane at an uncorrected threshold $p<.001$ with a cluster extent of 10 contiguous voxel.

We believe that our study has a number of strengths. We successfully recruited a unique cohort of HDGCs estimated to be more than 2 decades from motor onset and a well-matched cohort of control participants. We were able to demonstrate that our groups were not significantly different in tests traditionally sensitive to HD. We used a task with existing data in patients with $\mathrm{HD}$ and gene carriers closer to onset to allow us to better interpret our results, and we were able to replicate canonical results from the literature. Given both prior literature and our hypotheses, we restricted group comparison to a limited number of regions. ROls were defined using task data to identify voxels that showed hypothesis-relevant activity. Although task derived, a stringent statistical threshold was used to identify these clusters to limit the inclusion of false-positive voxels. These regions were identified based on shared activity across both groups and so were not biased to group differences. We also then ran a confirmatory analysis with a spherical $\mathrm{ROI}$ at independently reported coordinates and found the same group differences.

We also acknowledge that our study has the following limitations that readers may wish to consider. Our study design was cross-sectional and not powered to determine whether the effect that we found progresses as participants grow closer to disease onset. Although we lack longitudinal data, existing published HD data using this task may serve as an indicator of
A

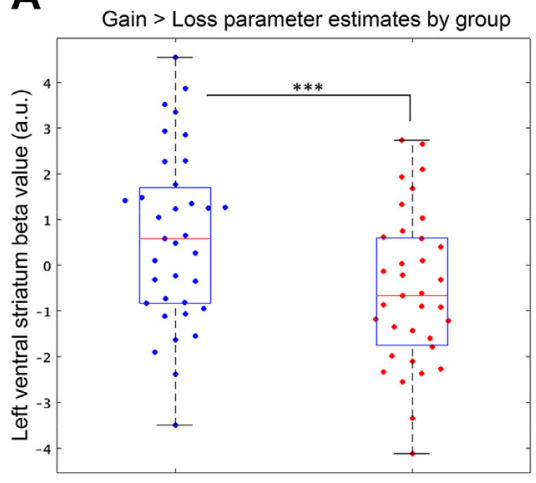

Gene Carriers
B

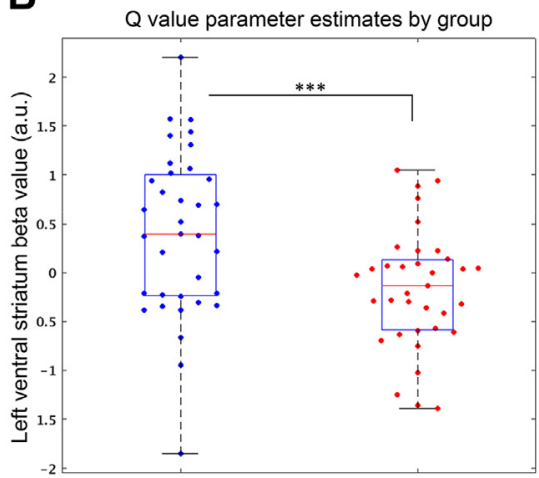

Gene Carriers
Figure 4. Left ventral striatum $\beta$ values for reward bias contrasts, by group. Individual left ventral striatum parameter estimates by subject in each group for (A) gain cue minus loss cue activity and (B) $Q$-value regressor. ${ }^{\star \star \star}$ Group difference, adjusting for effects of age, sex, and handedness, adjusted caudate volume, and depression scores, with significance of $p<.005$. a.u., arbitrary units. 
how task performance may change as the disease progresses. We also do not claim that our findings clearly represent disease per se; however, in conjunction with published data from the same task, we believe that our findings are likely to be antecedent of reward processing deficits that may emerge later. Recent work has shown the disease-related change particularly in fluid biomarkers can also be found at this point (38). Unlike previous work, we found differences only in fMRI data and not in behavioral data. Firstly, given that effect sizes for behavioral differences on cognitive tasks diminish in gene carriers further from disease onset, gross behavioral differences in our HDGCs may have been surprising (11). It is important to highlight, however, that lack of behavioral differences in this paradigm may also be related to task difficulty. Behavioral difference in reward learning may be seen this far from clinical onset with more challenging learning tasks.

\section{Conclusions}

Here, we demonstrate that healthy HDGCs approximately 25 years from motor onset show an exaggerated striatal response to gain-predicting cues compared with loss-predicting cues in a computational fMRI study. This difference between gene carriers and matched control subjects was also seen in striatal activity related to the predicted value of choice derived from a computational model. These results suggest that aberrant neural coding of valence and value may be one of the earliest features of carrying the HD gene, occurring decades before onset. These changes are not accompanied with robust behavioral changes, suggesting that an important window exists - where neural changes occur in HDGCs but before these changes drive potentially hard to recover behavioral impairment.

\section{ACKNOWLEDGMENTS AND DISCLOSURES}

This work was supported by the Leonard Wolfson Experimental Neurology Centre (Grant No. 525369 [to AN]) and by a Wellcome Trust Collaborative Award (Grant No. 200181/Z/15/Z). MRI scans for this study took place at The Wellcome Centre for Human Neuroimaging, which is supported by core funding from the Wellcome Trust (Grant No. 203147/Z/16/Z).

EJ, SG KO-C, PZ, JL, RS are all supported by a Wellcome Trust Collaborative Award (Grant No. 200181/Z/15/Z). MP is funded by a Human Biology fellowship from the Huntington's Disease Society of America (HDSA) and is supported by a Wellcome Trust Collaborative Award (Grant No. 200181/Z/15/Z). SP is supported by an ATIP-Avenir starting grant (Grant No. R16069JS), a Collaborative Research in Computational Neuroscience ANRNSF grant (Grant No. ANR-16-NEUC-0004), the Programme Emergence(s) de la Ville de Paris, the Fyssen foundation, and the Fondation Schlumberger pour l'Education et la Recherche (FSER). RBR is supported by a Medical Research Council Career Development Award (Grant No. MR/N02401X/1) and a NARSAD Young Investigator Grant from the Brain \& Behavior Research Foundation P\&S Fund. The Max Planck University College London Centre is a joint initiative supported by University College London and the Max Planck Society. The Wellcome Centre for Human Neuroimaging is supported by core funding from the Wellcome Trust (Grant No. 203147/Z/16/Z). GR receives grant funding from the Wellcome Trust. SJT receives grant funding for her research from the Medical Research Council UK, the Wellcome Trust, the Rosetrees Trust, Takeda Pharmaceuticals, NIHR North Thames Local Clinical Research Network, UK Dementia Research Institute, Wolfson Foundation for Neurodegeneration, and the CHDI Foundation.

AN designed the study, provided code for the fMRI experiment, obtained participant consent, performed neurological examinations, assisted with data collection, performed data analysis, and wrote the manuscript. EBJ collected imaging data, analyzed structural imaging and caudate volumes, and reviewed the manuscript. SG collected imaging data and reviewed the manuscript. KO-C performed out-of-scanner cognitive testing. PZ obtained participant consent and performed neurological examinations. RIS collected imaging data and reviewed the manuscript. $J L$ acted as the study manager and assisted in participant recruitment. MP provided valuable imaging analysis advice and acted as study manager. SP completed the previous study, provided valuable advice, supplied existing code for the previous experiment, and reviewed the manuscript. RR contributed to the study design and the coding of the experiment for $\mathrm{FMRI}$, provided valuable analysis guidance, and reviewed the manuscript. GR was a principal investigator for study, contributed to the design of the study, provided analysis guidance, and reviewed the manuscript. SJT was a principal investigator for the study, contributed to the design of the study, provided analysis guidance, and reviewed the manuscript.

We gratefully acknowledge the helpful discussions with Professors Trevor Robbins and Barbara Sahakian from Cambridge University. We also recognize valuable discussions with Dr. Bastien Blain, University College London, during analysis. We also recognize and would like to thank the participants for taking part in this study.

The authors report no biomedical financial interests or potential conflicts of interest.

\section{ARTICLE INFORMATION}

From the Huntington's Disease Centre (AN, EBJ, SG, KO-C, PZ, RIS, JL, MP, SJT); Max Planck University College London Centre for Computational Psychiatry and Ageing Research (AN, RBR); University College London Institute of Cognitive Neuroscience (RBR, GR); and Wellcome Centre for Human Neuroimaging (SJT), University College London Queen Square Institute of Neurology, University College London, London, United Kingdom; Laboratoire de Neurosciences Cognitives et Computationnelles (SP), Institut National de la Santé et de la Recherche Médicale; Département d'Etudes Cognitives (SP), Ecole Normale Supérieure; and Université de Paris Sciences et Lettres (SP), Paris, France.

Address correspondence to Sarah J. Tabrizi, Ph.D., at s.tabrizi@ucl.ac. uk.

Received Sep 29, 2020; revised and accepted Dec 14, 2020.

Supplementary material cited in this article is available online at https:// doi.org/10.1016/j.bpsc.2020.12.015.

\section{REFERENCES}

1. Ghosh R, Tabrizi SJ (2018): Clinical features of Huntington's Disease. In: Nóbrega C, Pereira de Almeida L, editors. Polyglutamine Disorders. Advances in Experimental Medicine and Biology, vol. 1049. Berlin, Germany: Springer, 1-28.

2. Duijn E Van, Craufurd D, Hubers AAM, Giltay EJ, Bonelli R, Rickards H, et al. (2014): Neuropsychiatric symptoms in a European Huntington's disease cohort (REGISTRY). J Neurol Neurosurg Psychiatry 85:14111418.

3. MacDonald ME, Ambrose CM, Duyao MP, Myers RH, Lin C, Srinidhi L, et al. (1993): A novel gene containing a trinucleotide repeat that is expanded and unstable on Huntington's disease chromosomes. Cell 72:971-983.

4. Bates GP, Dorsey R, Gusella JF, Hayden MR, Kay C, Leavitt BR, et al. (2015): Huntington disease. Nat Rev Dis Primers 1:1-21.

5. Langbehn DR, Hayden MR, Paulsen JS, PREDICT-HD Investigators of the Huntington Study Group (2010): CAG-repeat length and the age of onset in Huntington disease (HD): A review and validation study of statistical approaches. Am J Med Genet B Neuropsychiatr Genet 153B:397-408.

6. Tabrizi SJ, Scahill RI, Durr A, Roos RAC, Leavitt BR, Jones R, et al. (2011): Biological and clinical changes in premanifest and early stage Huntington's disease in the TRACK-HD study: The 12-month longitudinal analysis. Lancet Neurol 10:31-42.

7. Ross CA, Aylward EH, Wild EJ, Langbehn DR, Long JD, Warner JH, et al. (2014): Huntington disease: Natural history, biomarkers and prospects for therapeutics. Nat Rev Neurol 10:204-216.

8. Stout JC, Paulsen JS, Queller S, Solomon AC, Whitlock KB, Campbell JC, et al. (2011): Neurocognitive signs in prodromal Huntington disease. Neuropsychology 25:1-14. 
9. Tabrizi SJ, Leavitt BR, Landwehrmeyer GB, Wild EJ, Saft C, Barker RA, et al. (2019): Targeting Huntingtin expression in patients with Huntington's disease. N Engl J Med 380:2307-2316.

10. Wild EJ, Tabrizi SJ (2017): Therapies targeting DNA and RNA in Huntington's disease. Lancet Neurol 16:837-847.

11. Paulsen JS (2011): Cognitive impairment in Huntington disease: Diagnosis and treatment. Curr Neurol Neurosci Rep 11:474-483.

12. Dumas EM, van den Bogaard SJA, Middelkoop HAM, Roos RAC (2013): A review of cognition in Huntington's disease. Front Biosci (Schol Ed) 5:1-18.

13. Snowden JS (2017): The neuropsychology of Huntington's disease. Arch Clin Neuropsychol 32:876-887.

14. Waldvogel HJ, Kim EH, Tippett LJ, Vonsattel JG, Faull RLM (2015): The neuropathology of Huntington's disease. Curr Top Behav Neurosci 22:33-80.

15. Niccolini F, Politis M (2014): Neuroimaging in Huntington's disease. World J Radiol 6:301-312.

16. Calabresi P, Picconi B, Tozzi A, Ghiglieri V, Di Filippo M (2014): Direct and indirect pathways of basal ganglia: A critical reappraisal. Nat Neurosci 17:1022-1030.

17. Crittenden JR, Graybiel AM (2011): Basal ganglia disorders associated with imbalances in the striatal striosome and matrix compartments. Front Neuroanat 5:59.

18. Graybiel AM, Grafton ST (2015): The striatum: Where skills and habits meet. Cold Spring Harb Perspect Biol 7:a021691.

19. Haber SN (2011): Neuroanatomy of reward: A view from the ventral striatum. In: Gottfried JA, editor. Neurobiology of Sensation and Reward. Frontiers in Neuroscience. Boca Raton, FL: CRC Press/Taylor \& Francis, 235-262.

20. Haber SN (2016): Corticostriatal circuitry. Dialogues Clin Neurosci $18: 7-21$.

21. Wilson RP, Colizzi M, Bossong MG, Allen P, Kempton M, Bhattacharyya S (2018): The neural substrate of reward anticipation in health: A meta-analysis of fMRI findings in the monetary incentive delay task [published correction appears in Neuropsychol Rev 28: 507-508]. Neuropsychol Rev 28:496-506.

22. Nickchen K, Boehme R, del Mar Amador M, Hälbig TD, Dehnicke K, Panneck P, et al. (2016): Reversal learning reveals cognitive deficits and altered prediction error encoding in the ventral striatum in Huntington's disease. Brain Imaging Behav 11:1862-1872.

23. Palminteri S, Justo D, Jauffret C, Pavlicek B, Dauta A, Delmaire C, et al. (2012): Critical roles for anterior insula and dorsal striatum in punishment-based avoidance learning. Neuron 76:998-1009.

24. Enzi B, Edel MA, Lissek S, Peters S, Hoffmann R, Nicolas V, et al. (2012): Altered ventral striatal activation during reward and punishment processing in premanifest Huntington's disease: A functional magnetic resonance study. Exp Neurol 235:256-264.

25. Perry DC, Kramer JH (2015): Reward processing in neurodegenerative disease. Neurocase 21:120-133.

26. van Wouwe NC, Kanoff KE, Claassen DO, Richard Ridderinkhof K, Hedera P, Harrison MB, Wylie SA (2016): The allure of high-risk rewards in Huntington's disease. J Int Neuropsychol Soc 22:426-435.

27. Daniel R, Pollmann S (2014): A universal role of the ventral striatum in reward-based learning: Evidence from human studies. Neurobiol Learn Mem 114:90-100

28. Liu X, Hairston J, Schrier M, Fan J (2011): Common and distinct networks underlying reward valence and processing stages: A metaanalysis of functional neuroimaging studies. Neurosci Biobehav Rev 35:1219-1236.

29. Pessiglione M, Seymour B, Flandin G, Dolan RJ, Frith CD (2006): Dopamine-dependent prediction errors underpin reward-seeking behaviour in humans. Nature 442:1042-1045.
30. Joel D, Niv Y, Ruppin E (2002): Actor-critic models of the basal ganglia: New anatomical and computational perspectives. Neural Netw 15:535-547.

31. Collins AGE, Frank MJ (2014): Opponent actor learning (OpAL): Modeling interactive effects of striatal dopamine on reinforcement learning and choice incentive. Psychol Rev 121:337-366.

32. Franklin NT, Frank MJ (2015): A cholinergic feedback circuit to regulate striatal population uncertainty and optimize reinforcement learning. Elife 4:e12029.

33. Albin RL, Reiner A, Anderson KD, Dure LS, Handelin B, Balfour R, et al, (1992): Preferential loss of striato-external pallidal projection neurons in presymptomatic Huntington's disease. Ann Neurol 31:425-430.

34. Deng YP, Albin RL, Penney JB, Young AB, Anderson KD, Reiner A (2004): Differential loss of striatal projection systems in Huntington's disease: A quantitative immunohistochemical study. J Chem Neuroanat 27:143-164.

35. Zalocusky KA, Ramakrishnan C, Lerner TN, Davidson TJ, Knutson B, Deisseroth K (2016): Nucleus accumbens D2R cells signal prior outcomes and control risky decision-making. Nature 531:642-646.

36. Hikida T, Kimura K, Wada N, Funabiki K, Nakanishi S (2010): Distinct roles of synaptic transmission in direct and indirect striatal pathways to reward and aversive behavior. Neuron 66:896-907.

37. Hikida $\mathrm{T}$, Yawata S, Yamaguchi $\mathrm{T}$, Danjo $\mathrm{T}$, Sasaoka $\mathrm{T}$, Wang $\mathrm{Y}$, Nakanishi S (2013): Pathway-specific modulation of nucleus accumbens in reward and aversive behavior via selective transmitter receptors. Proc Natl Acad Sci U S A 110:342-347.

38. Scahill RI, Zeun P, Osborne-Crowley K, Johnson EB, Gregory S, Parker C, et al. (2020): Biological and clinical characteristics of gene carriers far from predicted onset in the Huntington's Disease Young Adult Study (HD-YAS): A cross-sectional analysis. Lancet Neurol 19:502-512.

39. Sutton RS, Barto AG (2012): Reinforcement Learning: An Introduction, 2nd ed. Cambridge, MA: Bradford Books/MIT Press.

40. Weiskopf N, Hutton C, Josephs O, Deichmann R (2006): Optimal EPI parameters for reduction of susceptibility-induced BOLD sensitivity losses: A whole-brain analysis at $3 \mathrm{~T}$ and $1.5 \mathrm{~T}$. Neuroimage 33:493504.

41. Rothkirch M, Tonn J, Köhler S, Sterzer P (2017): Neural mechanisms of reinforcement learning in unmedicated patients with major depressive disorder. Brain 140:1147-1157.

42. Wilson RC, Niv Y (2015): Is model fitting necessary for model-based fMRI? PLoS Comput Biol 11:e1004237.

43. Schonberg T, Daw ND, Joel D, O'Doherty JP (2007): Reinforcement Learning signals in the human striatum distinguish learners from nonlearners during reward-based decision making. J Neurosci 27:12860-12867.

44. Schmidt L, Braun EK, Wager TD, Shohamy D (2014): Mind matters: Placebo enhances reward learning in Parkinson's disease. Nat Neurosci 17:1793-1797.

45. Bora E, Velakoulis D, Walterfang M (2016): Social cognition in Huntington's disease: A meta-analysis. Behav Brain Res 297:131-140.

46. Henley SMD, Novak MJU, Frost C, King J, Tabrizi SJ, Warren JD (2012): Emotion recognition in Huntington's disease: A systematic review. Neurosci Biobehav Rev 36:237-253.

47. Chen JY, Wang EA, Cepeda C, Levine MS (2013): Dopamine imbalance in Huntington's disease: A mechanism for the lack of behavioral flexibility. Front Neurosci 7:114.

48. Voon V, Pessiglione M, Brezing C, Gallea C, Hubert H, Dolan RJ, Hallett M (2011): Mechanisms underlying dopamine-mediated reward bias in compulsive behaviors. Neuron 65:135-142. 